\title{
Inclusion of Adolescent Women in Microbicide Trials: A Public Health Imperative!
}

\author{
Suzanne Pomfret, Homerton University Hospital, London \\ Quarraisha Abdool Karim, Columbia University and CAPRISA, Durban, South Africa \\ Solomon R. Benatar*, University of Cape Town, South Africa and University of Toronto \\ *Corresponding author: Emeritus Professor of Medicine \& Director Bioethics Centre, Faculty of Health Sciences, University of Cape Town, E Floor, \\ Observatory, 7925 Western Cape, South Africa. Tel.: 27-21-406-6115; Email: Solomon.Benatar@uct.ac.za.
}

Conventional and well-established guidelines for the ethical conduct of clinical research are necessary but not sufficient for addressing research dilemmas related to public health research. There is a particular need for a public health ethics framework when, in the face of an epidemic, research is urgently needed to promote the common good. While there is limited experience in the use of a public health ethics framework, the value and potential of such an approach is increasingly being appreciated. Here we use two examples of adolescent women as potential candidates for participation in microbicide trials to illustrate how ethical decisions for public health research can be enhanced by drawing on both traditional research ethics guidance, and the emerging framework for public health ethics.

\section{Introduction}

Globally, one quarter of all HIV infections occur between the ages of 15-24 and more than half of new infections occur in people under 25 years (Macqueen and Karim, 2007). In 2004, there were an estimated 10.3 million people aged 15-24 with HIV infection worldwide, and an estimated 7000 new infections a day (Macqueen and Karim, 2007). The majority of these new infections are heterosexually acquired and in sub-Saharan Africa. In this region, HIV infection is about three-fold higher in young women compared to young men, and women are infected about 5-7 years earlier than men. Indeed the HIV epidemic in much of sub-Saharan Africa, especially southern Africa, is fuelled by high HIV incidence rates in young women under the age of 20 years. Several studies report that the HIV infection rate in teenage girls is 36 times that of boys in the same age group (Pettifor et al., 2005; Shisana et al., 2005). Therefore, reducing HIV infection in young girls under 18 years of age is critically important for altering the epidemic trajectories in this region, and for directly benefiting young women. This is undoubtedly a priority public health imperative.

Current methods for HIV prevention-abstinence, behaviour change, condom use and medical male circumcision-are likely to be of limited benefit for women especially in settings where gender power disparities contribute to excess HIV infection rates in women. The use of intravaginal microbicide gels and vaccines are examples of biomedical interventions under development that could offer some women some autonomy in reducing their vulnerability to HIV infection. Some indication of the potential public health impact of a 60 per cent efficacious microbicide can be gleaned from mathematical modelling data predicting that even if used by a small proportion of women in high HIV prevalence countries in half their sexual encounters, where condoms are not used, then 2.5 million HIV infections could be averted over 3 years (International Partnership for Microbicides, 2005). The inclusion of sexually active, HIV uninfected adolescent women (14-17 years of age) in HIV prevention efficacy trials will enable them to benefit immediately from successful trials as opposed to several years' delay for post-licensure testing and labelling for use in adolescent women.

Traditional drug development clinical trials start with Phase I safety testing, dose finding and pharmacokinetic studies in 10-20 normal subjects, then proceed to Phase II trials of about 50-80 persons with the disease where further dosing and administration data are obtained and a decision made on whether to proceed with larger studies. Phase III trials are then undertaken to establish effectiveness (depending on anticipated efficacy these could involve several hundred to several thousand participants). 
In contrast microbicide trials start with expanded Phase I trials of about 40-80 women at various levels of risk of HIV infection and then proceed to Phase III trials of several thousands of at-risk women to establish effectiveness. In the absence of a correlate of protection, HIV testing remains the best marker of both safety and efficacy. As the microbicide product pipeline increases, tough decisions need to be taken about selection of products to test in expensive Phase III trial designs. As we have learnt from the microbicide trials conducted to date, the current expanded Phase I testing strategies are insufficient to establish product safety or predict efficacy. Intermediate-sized trials (Phase IIb using several hundred randomized patients) have thus been used to establish safety and efficacy in an at risk population where incidence rates are sufficiently high and such studies could generate a more appropriate signal to proceed to expensive Phase III studies. With the shift to anti-retroviral-based products a more traditional drug development pathway is starting to emerge - that is with no intervening Phase IIb studies.

To date, including recently terminated and ongoing microbicide effectiveness (Phase III) trials, over 22,000 women have been enrolled in numerous safety and efficacy trials. By mid-2006, five candidate microbicides were being tested for efficacy (Phase III trials) to prevent HIV infection at more than 35 clinical sites in 17 countries. More than half a dozen other products are being assessed in early safety (Phase I) and expanded safety (Phase IIb) trials (Alliance for Microbicide Development, 2008). To date only the Carraguard trial (SkolerKarpoff et al., 2008) enrolled 16- and 17-year-old women.

Given the public health imperative for enhancing protection against infection in young women and the need for data on the safety and effectiveness of microbicides in adolescents as a potentially very effective future public health tool, we contend that it may amount to public health negligence not to include women under the age of 18 years in Phase III trials

The recent early closure of several microbicide and vaccine trials illustrates that consideration of safety is not a trivial issue when testing experimental products. Traditional ethical considerations of autonomous decisionmaking and protection of vulnerable populations are of great social value. On the other hand, where the public health imperatives are of the magnitude being experienced by young women in sub-Saharan Africa, it is necessary to consider newer approaches more focused on public health and the common good that could assist in moving forward.

It could be argued that valid concerns regarding protection of vulnerable minors are perhaps being overemphasised and insufficient attention is being paid to the fact that adolescents are likely to benefit most from an efficacious microbicide, and that a very important social good would be served by establishing an effective and safe microbicide for use by young women. Moral and judgemental positions on sexual activity in young women (including outside of marriage), concerns about cognitive ability to provide first-person consent, ability to adhere to complex trial procedures, potential risk to immature genital tracts and safety concerns relating to exposure to experimental drugs, all of which have been cited as reasons for not enrolling adolescent women in microbicide trials, despite the elusive factual basis for all of these concerns, are blocking progress. These concerns need to be dealt with to improve the feasibility of accruing cohorts of adolescents, the ability to retain these cohorts and to obtain adherence to study procedures such that the power and scientific integrity of the trial is not compromised

There is potential for risk of HIV infection in all trials and while steps are taken to minimize this (education about use of condoms and avoidance of multiple partners), risk cannot be eliminated in the absence of a proxy correlate of protection. Indeed it is in clinical trials inclusive of at-risk populations that have been most predictive of harm or potential harm which earlier safety studies in no, or low, risk populations did not identify. Early microbicide trials of first- and second-generation microbicides were less specifically HIV protective compared to new generation microbicides that are anti-retroviral based. At this point in time HIV testing is both a safety and an efficacy marker and, given the topical application of microbicides safety assessments that include genital examinations are essential. By definition microbicide trials only include women who are sexually active. HIV testing for safety and efficacy monitoring with ARV-based products usually involves monthly HIV testing. Safety monitoring using laboratory markers, such as tests of renal and liver function, and pelvic examinations, is undertaken less frequently-in some instances once in 3 months. The comprehension of information provided during the process of obtaining consent is even more critical in the conduct of trials that include adolescents and it is necessary to ensure that there is sufficient cognitive ability to consent, with comprehension assessed prior to initiation of study procedures and on an ongoing basis.

\section{Cases for Consideration}

We shall consider here why it is important for adolescents, such as the two in the case studies below, to be considered for inclusion in Phase IIb or Phase III microbicide trials, concentrating on the HIV risk that is relevant for these women. 


\section{Case 1}

A 16-year-old sexually active girl wishes to participate in a microbicide study. She is not able to give legal consent as she is a minor but she does not want her parents to know that she is participating.

\section{Case 2}

A 16-year-old sexually active girl wishes to participate in a microbicide study. She is not able to give her legal consent as she is a minor. She is unaware of who her father is as she has been raised by her mother. Her mother has recently died from AIDS and she is the head of the household and taking care of her siblings.

These two case studies focus on whether adolescents can give valid consent for their participation in a clinical trial and on whether good arguments, based on public health considerations, can be provided for their inclusion even if conventional criteria for informed consent cannot be fully met. One of the adolescents we discuss has parents, whom she does not want to have knowledge of her participation, and the other is the 'head of the household', so already fulfilling an 'adult' role. Using the conventional approach to research ethics, these young women could not be included in microbicide trials without parental or guardian consent.

We shall argue that conventional ethical guidelines are inadequate for the ethical dilemmas that must be faced when the common good is at stake in a pandemic that is costing millions of lives, and that where potentially effective preventive measures must be researched there is scope for inclusion of a public health ethics approach that could provide justification for including adolescents in trials with major public health benefits for their age group. We note that throughout this discussion, we are referring to adolescent participation in Phase IIb/III Microbicide trials. The three prerequisites for enrolment in such a trial are a negative HIV test, no desire or intention to become pregnant during the trial, adherence to study procedures including product use, monthly HIV and pregnancy testing and several pelvic examinations.

\section{An Outline of a Public Health Ethics Approach}

A public health ethics framework for considering ethical issues is one that allows for consideration of the common good in addition to individual choice and rights. While there is as no coherent framework for a public health ethics approach that has been accepted or tested, several authors have offered contributions to how we should think about public health care dilemmas.

Roberts and Reich have noted that when making public health decisions, one of the most difficult issues is the need to balance what is considered to be best for the population while retaining protection of individuals (Roberts and Reich, 2002). In their review they describe how ethical arguments in public health can be grouped into three main categories, each representing a major theme in contemporary public health discourse: utilitarianism, liberalism and communitarianism. They note that while utilitarianism is highly influential in public health decision-making, liberalism also has considerable force as evidenced in the approach to patients who are HIV positive and that the communitarian perspective, with its focus on creating a good society in which individuals within that society exhibit virtues appropriate to that society, also offers ethical guidance (Roberts and Reich, 2002).

Public health ethics debates embrace arguments across these philosophical domains each of which may dominate in specific situations, but what is important is that there should be an explicit discussion of the issues and well-constructed arguments that facilitate movement towards a coherent position.

Several operational frameworks and principles have been offered for the analysis of ethical issues in public health practice. Nancy Kass (2001) has suggested that six primary questions should be addressed in relation to the ethical dimensions of any proposed public health program:

- What are the public health goals of the proposed program?

- How effective is the program in achieving its stated goals?

- What are the known or potential burdens of the program?

- Can burdens be minimized? Are there alternative approaches?

- Is the program implemented fairly?

- How can the benefits and burdens of the program be fairly balanced?

Childress and colleagues (2002) enumerate five considerations to be weighed when analyzing the ethical dimensions of public health action: effectiveness, proportionality, necessity, least infringement and public justification (Childress et al., 2002). Upshur (2002) has added to these in his proposal of the following four principles to guide the justification of public health intervention: the harm principle; the principle of least restrictive or coercive 
means; the reciprocity principle; and the transparency principle.

The seven emerging key principles that could constitute a public health ethics framework are listed below (Childress et al., 2002; Upshur, 2002):

- Transparency Principle: Any public health decision has to be as clear and accountable as possible, as well as free from political interference.

- Effectiveness Principle: Requires evidence of the effectiveness of a management in improving public health, if other moral considerations are to be infringed upon.

- Necessity Principle : No other method of achieving a particular 'end' would have less conflict with other moral considerations.

- Proportionality Principle: A positive balance should be achieved between potential benefits of a public health intervention and any adverse effects brought about by a conflict with other moral considerations, such as infringing upon individual human rights.

- Harm Principle: The only justification of restricting the liberty of an individual/group is to prevent harm to others.

- Reciprocity Principle: The state should provide appropriate assistance to individuals to facilitate their public responsibility to adhere to any Public Health intervention, for example, treatment or quarantine.

- Least Restrictive Means Principle : Any less coercive means must be tried first before it is justified to implement more intrusive Public Health restrictions on liberty.

The ideas presented above have not yet been extensively debated for their strengths and limitations. How and when they will be implemented and with what effects will be determined by an ongoing debate that will foster refined concepts and their application.

We suggest that where consideration of some balancing between individual rights and the common good is required for the implementation of much needed, and potentially very beneficial, public health interventions, including research to provide the evidence for such interventions, a public health ethics approach supplementing traditional ethical principles could facilitate 'trade-offs' that have a morally convincing rationale.

In this paper we illustrate how the application of the principles of public health ethics may be used to motivate the inclusion of adolescent women in HIV prevention trials of experimental interventions such as vaccines and microbicides, without neglect of the conventional principles considered in human research. In addition we propose an additional principle-the common good principle-for consideration when public health is significantly threatened.
- Common good principle — during a public health crisis in which many lives are threatened, and only inadequate preventive measures are available, the common good can be elevated above the good of individuals for researching or implementing a potentially effective preventive method.

This principle should not be used as a stand-alone principle, but rather within the emerging framework of public health ethics and without losing sight of the principles of conventional research ethics.

It should be noted at this stage that the principles of public health ethics listed above were initially formulated with other situations in mind-for example dealing with the ethical issues that arise in relation to containing spread of infectious disease. They were not formulated with public health research ethics dilemmas in mind. However, HIV prevention research presents the potential for great public health benefits, so we consider it appropriate to explore how these newly evolving principles may be applied in such a context. It should also be noted that these are all prima facie principles and that their ranking is influenced by the circumstances in which they are applied.

\section{Including Adolescents in Microbicide Trials}

There are three distinct issues to be considered in relation to inclusion or exclusion of adolescent women in microbicide trials:

- The need for data on the safety and effectiveness of microbicides in adolescents, as a potentially very effective future public health tool.

- Traditional protection of vulnerable minors.

- Some other ethical considerations including recognition of the cognitive autonomy of adolescents.

At an anatomical level, cervixes of younger women are not yet fully mature. The columnar epithelium of part of the outer exposed part of the adolescent cervix has yet to change to the more protective squamous epithelium, making young women more susceptible to STDs. Up to 80 per cent of teenagers will have less progesterone within 4 years of menarche, as they have anovulatory cycles (Global Campaign for Microbicides, 2005). This difference in progesterone levels may have a significant effect on the vagina's local immune responses.

\section{Necessity}

The 'Necessity' principle demands that no other method of achieving a particular 'end' (in this case protection 
from infection) would have less conflict with other moral values.

It could be argued that rather than include adolescents in microbicide trials more could be done to educate individuals, and promote condom use, but the reality is that more than this is needed to curtail the pandemic as sequential HIV/AIDS statistics show. An effective microbicide would be a simple intervention that could help, in particular as it promotes empowerment of women as a good example of a public health intervention that also promotes individual autonomy, and therefore adolescent inclusion would be permitted under this principle.

Microbicide use is initiated by women in circumstances where they are aware of the risk of HIV infection but are unable to get their male partner to use condoms. Microbicide use will enable women to take at least some personal control of their HIV risk. In relationships characterised by good partner communication, including communication about HIV risk and risk reduction, it becomes a matter of choice for women to use the microbicide. Given the substantial threat that HIV poses to women's survival the availability of a method that they can initiate to reduce their HIV risk and enhance survival cannot be underestimated. In the case of adolescents, HIV-free survival in the teens will facilitate them entering their twenties with greater social and cognitive ability to negotiate HIV risk reduction.

Notwithstanding age differences in cohorts, we are learning from completed microbicide and vaccine trials, that safety data generated in Phase I and II trials are not adequate predictors of longer term safety of product with extended use as is the case with Phase III trials. In the cases we are considering here, these girls are being considered for participation in a Phase III trial, with the presumption that Phase I and II trials have been carried out only in adults.

A crucial point in defence of a decision to include high-risk adolescents in microbicide trials is that it increases the potential for such trials to show the efficacy of this potentially important public health measure (Kock et al., 2006). High-risk adolescents include those living in countries where HIV is primarily sexually transmitted and where the HIV prevalence and incidence rates are high. This is the case for young women in sub-Saharan Africa where HIV infection is synonymous with sexual debut and HIV infection is 3-6-fold higher compared to males in the same age range. It would be pointless to study a preventative intervention, such as microbicides in a low-risk population. Once efficacy has been demonstrated it will then be necessary to show that microbicides are effective outside of trial conditions and in the realworld setting. Negative studies are also of value as long as trial participants have not been put at any greater risk.
Taking these factors into account, Moscicki (Division of Adolescent Medicine at the University of California, San Francisco, as reported by the Global Campaign for Microbicides) has expressed the view that the biologic and behavioural differences between young female adolescents and older women justify separate safety and effectiveness data on adolescents (Global Campaign for Microbicides, 2005). This invokes the principles of necessity in including adolescents in microbicide trials, and the potential effectiveness of the intervention.

\section{Effectiveness}

The 'Effectiveness' principle emphasizes the importance of evidence. The problem is that we have a paucity of evidence regarding the risks and benefits of microbicide use in adults or adolescents. Indeed we may never know the risks, if microbicides are not tested on the adolescent age group. If the efficacy of a microbicide were to be shown in a good trial, then obviously its use as a public health intervention would not be contested, although effectiveness studies in real-world situations would still need to be conducted. However, here we are discussing adolescent involvement in the trial when we lack the evidence required for invoking this principle. Again, however, the chicken-and-egg situation arises-we need a trial to establish the risks. The solution may be to involve teenagers in Phase I and II trials with parental permission in the hope that this may be sufficient to satisfy this principle. However, as we are considering minors being exposed to unknown risks, we acknowledge that there is indeed difficulty in meeting the requirements of this principle.

\section{Common Good}

Few would disagree that a method of preventing HIV infection among young women, that is effective in realworld settings, would be a considerable advance in reducing the impact of a pandemic that is causing major loss of life in young people. Given the need for solidarity in the face of common threats it seems entirely reasonable to give a high priority to the principle of the common good in deciding whether to include adolescents in microbicide trials.

\section{Traditional Protection of Vulnerable Minors}

Traditional protection of vulnerable minors has been focused on avoiding research on them that could be done on others, obtaining parental or other appropriate 
surrogate consent, and through obtaining assent from minors. We demand parental consent for minors simply because minors are thought to lack the full capacity to understand everything that needs to be understood to make an informed decision. It is not doubted that they may know what they want, and that they can make a decision. The pertinent question is whether or not such a decision is an adequately informed and considered one. It could be argued that some parents may make a worse decision for a particular individual, but no system can ever be fully foolproof. Parents in all societies are considered to have responsibility for the best interests of their child all their life, and so they are the obvious proxy decision makers. It is often hard to agree with a decision if it is not one that we ourselves would have made, but the whole point of respecting an informed decision is that there is a choice to be made and we should respect the decision of a person empowered with enough information to make the best decision for themselves. If we were all to make the same choice then any decision-making process for such a choice would be totally futile.

Much attention has been focused on the conduct of HIV prevention trials to ensure that obtaining informed consent is not merely procedural in terms of a signature obtained prior to study procedures being initiated. Indeed, novel and innovative approaches have been developed to ensure that participation is informed and truly voluntary. Efforts have also been promoted to ensure that all participants are fully informed about randomisation and uncertainty of product efficacy in addition to receiving adequate counselling on known methods of HIV prevention (Global Campaign for Microbicides, 2005). Comprehension assessments following enrolment consent and prior to randomisation have become the norm in HIV prevention trials.

These steps ensure the 'consentee's' decision-making capacity, i.e., their competence, and their comprehension, as well as an explicit declaration to participate or refuse. These points are particularly relevant in relation to considering adolescents' autonomous consent or the consent of parents. The problem faced in the cases under consideration is that the adolescents wish to participate either without parental consent, or in the absence of parents to consent for her.

It is understandable for the first 16-year-old not to want her parents to know that she is taking part in the trial. First, she may not want them to know that she is sexually active. Her parents may not approve, their reactions would be unpredictable, and anyway this should not be relevant if personal choice (regardless of legal age) has high moral value. We also have to consider the consequences of requiring and obtaining parental permission when adolescents are found to be HIV positive. Some may argue that any infringement on the confidentiality entitled to an individual under these circumstances is unethical, given that the stigma involved in assumed HIV infection is the same as for actual HIV infection. However, the harm principle would allow breaking confidentiality if this would prevent harm to identifiable others.

In the absence of parents it may be necessary to consider the question of community consent, which may involve the consent of a village chief or church leader. Partner consent may also need to be considered in some contexts. It is often the cultural norm in the developing world to grant either initial or total authority for decision making to the male in relationships. It seems paradoxical to consider men's involvement, much less their consent, to test a product that could allow disempowered, and often scared, women to protect, and empower, themselves, especially as many men refuse to use condoms, and levels of domestic violence are often very high. This consideration limits a female participant's autonomy and it seems common sense for it to be up to the female participants to determine if and how their male partners are to be involved. A full discussion of these matters, and of how women could be empowered to refuse sex, is beyond the scope of this essay, but these issues do need to be considered as they have some bearing (albeit small) on both the cases we discuss.

It should be acknowledged that the success of any possible future for microbicides relies on their acceptability to men. The same can be said for users' adherence to regular use. In South Africa, the principal worry for men about microbicide use is that it may interfere with their sexual pleasure. Some traditional laws would go so far as to punish a woman, and probably readily force her not to participate, if she is found by her partner to be using 'something in her vagina' without his knowledge (Global Campaign for Microbicides, 2005: chapter 5, 24).

\section{Harm}

The standard way of talking about harm is to say that the only justification for an intervention that limits individual liberties is that the intervention must prevent harm to others. This is not directly applicable here as allowing an adolescent to consent to participation is not an infringement of liberty, unless it is viewed as interfering with her liberty not to be an under-age research subject. From the perspective of the common good principle it can be argued that if an adolescent did not take part in such a study, harm could be done to her sexual partners and any children she may bear, or in the case of 
the second girl to those for whom she is now responsible. It would also allow the interpretation that reciprocal freedom from harm (within consideration of the common good) would support participation of adolescents in socially valuable research.

\section{Some Other Ethical Considerations}

\section{Autonomy}

It is probable that adolescents would want to take part in microbicide and vaccine trials and obviously it is important for any such potentially preventive measure to target young people before their sexual debut. In support of this contention we note that Jaspan et al. found that 79 per cent of adolescents aged 11-19 years in a Xhosa-speaking community near Cape Town were willing to participate in a vaccine trial (Jaspan et al., 2006).

\section{Recognition of Adolescent Cognitive Autonomy}

Although cognitive, abstract thinking is developed in mid-adolescence (14-16 years), the age range during which such thinking is adequately developed is very wide. We need to ask whether the cognitive abilities of the majority of adolescents may approximate those of young adults, giving them the ability to understand the longterm risks and benefits of any research. With the priority many governments are placed on schooling in much of the developing world, it is likely that many adolescents may have had more formal education than many adult participants in their countries, and with that, more training in reasoning and rational thinking. Both these points are relevant to informed consent.

The cases we are considering here resemble the case in which the notion of Gillick/Fraser competence was explored (Wheeler, 2006). From this it can be argued that if a minor can be shown to be mature enough to understand the purpose of the proposed study and the level of involvement requested, including benefits and risks, then he/she should be able to consent. However, this may need to be determined by two professionals (as for Gillick/Fraser competence) who are independent from the researchers in order to avoid bias and to provide as objective a judgement as possible.

It is of interest to note that in South Africa adolescents, 16 years and over, can legally consent to sex, but are not permitted the autonomy to legally consent to participation in a trial designed to help protect them during sex. The Children's Bill in South Africa (June 2005) states that young people can have access to contraception without parental consent from the age of 12 (Kock et al., 2006). The new Health Act states that children as young as 12 can consent to medical procedures if they can be shown to understand fully the process and consequences. Yet 16-year-olds cannot legally consent for themselves to participate in trials evaluating preventive measures. Discussions held with the Medicines Control Council (MCC) and Ethics Committees in South Africa has led to a better appreciation by IRBs of HIV risk faced by adolescent girls. Indeed one of the co-authors ( $\mathrm{Q}$. A. K.) has had the experience of MCC and UKZN Research Ethics Committees requesting an explanation for why 16- and 17-year-old women were not included in a microbicide trial being conducted by her.

In a study of the informed consent process, as viewed by children, it was found during post-test counselling that concrete elements of research, such as the duration of the study and benefits to themselves, were understood the best. However, participants had difficulty understanding more abstract issues such as the purpose of study, benefits to others and alternative treatments. While the study included children who had yet to reach the age for mature abstract thinking the authors also attributed the lack of understanding to inability to place their participation in context because of lack of life experience (Susman et al., 1992).

\section{Some Implications of Adolescent Consent}

Potential repercussions of excluding parental consent include the possibility that if parents were to find out that some teenagers in the community were involved in such a trial and suspect that their children are involved, researchers could be alienated from communities and lose support for the study, with consequent loss of many benefits that could accrue to communities from the research. In order to avoid this, consideration could be given to asking the community to give permission for teenagers to participate and then asking the teenagers themselves to give personal consent without having to divulge their decision. However, even this may lead to pressure from parents or friends to reveal who is involved, and participation may have repercussions for the youth.

There is concern too about infringing upon the rights of parents as in addition to their natural responsibility they also have a legal responsibility to care for the welfare of their children. However, precedents have been set regarding bypassing of parents' wishes in situations where public health considerations are overriding.

Singh et al. writing about the importance of enrolling adolescents in trials such as these, note that The Human Sciences Research Council reports an almost doubling of South African households that are headed by children, 
or consisting only of children, in 2002-2005 (Singh et al., 2006). In 2005, among children aged $12-18$ years, 2.6 per cent $(180,433)$ identified themselves as head of households. Among orphans, 2.8 per cent $(213,859)$ identified themselves as being head of households, and almost 65 per cent of orphans live in community/family care or child-headed households (Singh et al., 2006). The girl in Case Study 2 is thus far from an isolated case and this position should be acknowledged for legal and medical purposes that would hopefully include the ability to participate in trials.

It is notable too that many adolescents who attend prenatal and family planning clinics do not live with their parents. They either live alone because their parents are deceased, live/work in a distant area or because they are in the care of members of the community, usually relatives (with most of these surrogate caregivers not formally appointed or recognised as the child's legal guardian) (Singh et al., 2006). Under these circumstance it seems to be most logical for the adolescent themselves to give consent if they can be shown to have the requisite understanding. It could be argued that the very fact that they are attending either a family planning clinic or antenatal clinic identifies them as probable mature minors.

It is perhaps easier to argue for the inclusion of the second adolescent in a trial. However, it would be necessary to indicate whether it would be fair to allow her to participate and not the other adolescent? Her situation is very unfortunate but does this warrant her to be suddenly assigned 'adult' status. We are told that she has the responsibility of an 'adult' position, but we have no idea how she is coping with such responsibility ahead of her years. Her position now as 'head of the household' does not necessarily make her capable of making an informed choice. She may have greater experience of some aspects of life than the first girl, but does that necessarily make her better equipped for such a decision?

Conversely, if the situation remains that adolescents can only participate, with parental consent, then, this would also be unfair, as it would exclude the second girl because of her situation, as she has no parents to consent for her. One could also argue, hypothetically speaking, that she may be at more risk. The financial pressures on this girl could be so heightened that she is more ready to take part in 'transactional sex' with older men, both factors putting her at an increased risk.

A further issue is that in many countries sexual intercourse, even if consensual, below certain ages is considered rape and there may be legal obligations of researchers to report rape. However, there have been several precedents where research has been conducted in vulnerable populations in which sexual activity, injected drug use and same-sex relationships are considered illegal, without obligation to report these individuals to law enforcing agencies on the grounds that the relationship between research participants and the study team is a confidential one and needs to be respected. The common good principle could also be invoked here to support research designed to benefit whole groups. Adolescent and adult women participants who report sexual, verbal, or physical abuse are counselled about their rights, informed about what legal recourse is open to them and directed to sources of support in the community that they can access.

In the case of sexually active adolescents it is not for the researcher to pass moral or other judgement on the adolescent being sexually active. The researchers' responsibility in undertaking such research is to minimize harm including risk of acquiring HIV infection. The reality is that despite legal restrictions many adolescent girls and boys are sexually active and not all of the coital activity is coerced but is in fact consensual.

\section{Beneficence}

Well-intended advantages of participation in microbicide trials include:

- trial-provided condoms and condom counselling in the participant's own language-an important public health advantage for everyone

- decreased risk of HIV infection for all regardless of which arm of the trial to which they are randomised

- material benefits, which include reimbursement for transport time and childcare, and food at the clinic

- early detection and treatment for STDs, discovered during the trial

- HIV counselling and referral for both the participant and their partners to suitable care and/or referral for any pregnancies during the trial.

While these may be viewed as inducements to participate, it could also be the case that they would not be seen in this light in the face of what is probably minimal risk and potentially significant benefits.

There are also many collective benefits (common good) that should ensue for the local communities when a trial is conducted, some of which are immediate and tangible while others manifest later:

- health services are improved in the locale of the trial in order for trial 'managers' to maintain an appropriate standard of care - whatever that is decided to be in a particular context (Shapiro and Benatar, 2005) ${ }^{1}$

- enhanced access to tests and information and training 
- fewer HIV seroconversions in women in the general community because of the decreased risk brought about by the efforts offered as part of the trial (Global Campaign for Microbicides, 2005).

- access to antiretroviral treatment in the future

- overall benefit for a community that feels it is 'doing its bit' for the pandemic - so generating feelings of self-worth and hence community well being.

\section{Justice}

Within bioethics debates the principles of autonomy (respect for persons) and beneficence are now increasingly linked to consideration of the principle of justice. Expansion of microbicide research to include adolescents with their personal consent would be supported by the principle of justice, as such inclusion would facilitate the equitable spread of the benefits of research and thus hopefully, improve health for many - an important aim for public health interventions.

However, it is the burdens of research that are problematic here. It could be argued simply that minors, including adolescents, are a vulnerable group, and as such should not be party to more burdens/risks than occur simply as a course of nature. This would justify providing them with benefits while not requiring them to share in the risks. This is in general the approach we take with children on the assumption that when they become adults they will then bear some of the burdens required to assist new generations of children. Such an approach may, however, delay the acquisition of data required to advance the health of adolescents

Debates on justice in research in the 1980 s provide a precedent. At that time the idea that women were vulnerable and not allowed to take part in research, was replaced with the argument that ethically they had to be included so that they could enjoy the benefits of research, as well as share in the risks, especially when the research was particularly relevant to their health (Epstein, 2007). We suggest that the same could apply to adolescents under the conditions we have described here.

In order to minimise the risks involved for adolescents, a good compromise would be to first conduct Phase I and II trials involving willing adolescents who have parental consent. Then, when more information is available about their safety and communities are more comfortable with the knowledge of these, Phase III trials could be set up on the basis of community collective consent followed by individual consent given by the adolescent if she can be shown to understand the process and consequences adequately, and this is agreed on by two objective professionals. This would add support for the argument for participation under the 'Proportionality' principle.

\section{Proportionality}

The 'Proportionality' principle requires that there be a positive balance between potential benefits of a public health intervention and the adverse effects of infringing upon an individual's human rights. This is especially relevant to this discussion. It also must be expanded to encompass a broader balance of benefits and risks, because any public health intervention must simply have greater benefits then risks. We have discussed the benefits for participation for both the adolescent and the community, and we suggest that it is also necessary to emphasise benefits to the host country and therefore to public health in its broadest sense.

Potential harms include adverse physical effects of microbicide, stigma, emotional stress consequent upon participation or outcomes of the trial and the adverse effects of an adolescent participating without parental consent. Consideration of this principle also requires balancing infringement of parental rights to protect their children, against the public health benefits of adolescent participation. However, it is also important to consider the individual benefits to the adolescent, including respect for her autonomy and decision-making capacity. Such individual benefits do after all contribute to public health benefits, and need to be considered in the proportionality equation.

\section{Transparency}

Trials involving Tenofovir highlighted the importance of the 'Transparency' principle in building community trust (Anonymous, 2005). The crucial element here is that in all trials there should be as much transparency as possible and promotion of as much understanding as possible at community level, to ensure open accountability, legitimate participation and minimal risks. In a microbicide trial involving adolescents, the above-mentioned Phase I and II trials would be part of the process of promoting transparency

\section{Reciprocity}

This principle requires that individuals who contribute to the public good at the expense of their own wishes or rights (or without parental consent) should receive the support required from the state to enable them to make these sacrifices more easily. In relation to microbicide trials this principle demands that future responsibilities be clarified so that provision of care when needed is met. Adolescent participation would therefore require consideration of post-trial access. Unless such issues are dealt with proactively, ethical quandaries could delay or 
derail a generation of future trials (Global Campaign for Microbicides, 2005).

Obligations to adolescents would be the same as to adults, with recognition that it is increasingly accepted that becoming HIV positive during a trial imposes the obligation on researchers to ensure ARV treatment when this may be required. The difficulty lies in meeting this obligation when the need may only arise a decade or more after the trial has ended

With this in mind, several operational issues would need to be addressed-namely, the mobility of the adolescent population and subsequent participant tracking difficulties; future access to, and 'marketing' of, microbicides for vulnerable populations; both provision of and fiscal considerations of future access to ARVs for those participants who contract HIV during the trial and, finally, how continued access to microbicides (if deemed effective) would be maintained, both logistically and financially. It is difficult to properly consider a minor taking a personal risk when the answers to these questions are not available. If we were to consider that adolescents should participate, especially under the justification of advancing public health, then the risk should be as small as possible-which will only be the case, if the above questions are answered —or the public health advantages so great that these become of major significance.

\section{Some Cultural Considerations}

Molyneux et al. emphasise that, in such discussions of ethics there is an underlying controversy regarding whether conceptions of human rights and models of ethical reasoning are absolute in their application to communities and countries that differ enormously in socioeconomics, culture and politics (Molyneux et al., 2005). They challenge the notion of individual consent as far as relevance and priority go and explain this by referring to the low levels of formal education; poor access to good quality biomedical services; different values, priorities and understandings of health and illness; the roles of the community, chiefs, sangomas or alike, elders, men and women and, lastly, the problems of the actual physical act of reading and signing the consent form. They acknowledge the importance of community-level consultation and permission, and alternative methods of communicating other than reading and writing. From their work in Kenyan communities they unequivocally observed that for children aged less then 18 years, parents were asked (and probably should be in that society), and that in general it was the father who had the stated decision-making authority. If the father was absent, or could not give permission, then the mother, or other males or elders of the household, could do so. We cannot necessarily extrapolate this to all communities but it does give us an idea that what we argue for may not necessarily be the same in communities far removed from our own. As Upshur says, '... populations are constituted by diverse communities of heterogeneous beliefs and practices ... these may at times conflict' (Upshur, 2002).

\section{A Balanced Decision}

The value of respect for persons and informed consent would make it incumbent on researchers to do everything possible to ensure that the moral requirements for informed consent are met even if the legal (age) requirements cannot be met. Considerations of inclusion as justice would favour adolescent participation. The public health necessity for the knowledge that could be acquired from including adolescents in microbicide trials, the relatively minimal harm that would be done to them, the benefits that would flow to adolescents as a group and the reciprocity value associated with guaranteed post-trial access would all be in favour of including adolescents in the microbicide trials. We conclude that there is adequate moral justification for doing so utilising both conventional concepts of research ethics and newly formulated principles of public health ethics.

\section{Conclusions}

The two cases we discuss raise a very realistic debate on how to consider public health goals when these may infringe on individual rights. We have noted that there are both benefits and disadvantages of the permissive inclusion of adolescents in a microbicide trial. The public health debate centres on the balance between the rights of the individual to be treated equally and fairly, and, the need to achieve the greatest potential public health benefits (Benatar, 2006). An emphasis on Public Health is far from misplaced and of ever-increasing importance as the growing HIV/AIDS pandemic so clearly illustrates.

The health of whole populations is, in part, made up of the sum of every individual's health and therefore individual health does have a bearing on population health. However, public health is more than just an aggregate of the health of many individuals. Public health considerations require that we also address the socio-economic and cultural foundations of health, and their influence on inequalities (Benatar, 1998; Birn, 2005). Public health aims to protect those who need protection, and hence has a high regard for the human rights that at times it has to infringe. 
There is also a need to challenge conventional public thinking and complacency. Nixon and colleagues refer to the magnitude of the response that was mounted against SARS, a global infectious disease that predominantly affected the rich world, and compared this to the inadequate response that is being mounted against infectious disease such as HIV and TB, which affect the developing world predominantly but kill over 10,000 times as many people every day than SARS did during the 2002-2003 epidemic (Nixon et al., 2005). When we think of public health we often think of extreme, paternalistic, intervention measures. The recent SARS problem in Canada led to quarantining - a huge infringement of individual liberties for the good of the public (Singer et al., 2003). Such interventions could not be condoned for HIV for many reasons. However, it is now necessary to consider ethical justification for public health measures that could significantly impact on this tragic HIV/AIDS pandemic.

An effective microbicide could not only make a significant contribution to public health, but it would also promote individual health. This is an example of not relying on paternalism but rather on empowering individuals to be autonomous and making their own choices. It stands as an excellent example that one good need not negate another good and that there is a symbiotic relationship within which promotion of either individual health or public health can lead to the promotion of the other.

As consideration of adolescent participation in microbicide trials will inevitably be associated with potential adverse effects, decisions to involve them will be controversial. Their inclusion can be defended using principles of public health ethics combined with conventional individual-based ethical reasoning.

To be ethical and responsible requires consideration of the public health effects of our actions. This applies especially to health professionals, all of whom face limitation on use of resources, although admittedly to very different extents in different countries.

Any possible future effective microbicide would be a highly valued public health tool, and therefore there is a need to support microbicide trials with inclusion of those groups that are at the highest risk-here, adolescents-who also stand to be the most significant beneficiaries. Participation in such trials is a perfect example of maximising public health at many levels - the possibility of finding an effective HIV preventative measure, producing community health benefits as well as benefits for the host country and participants themselves.

To achieve these goals requires a 'shift in mindset away from strong individualism towards respect for individuals within a context of a sense of duty towards a com- munity' (Benatar et al., 2003). Generally, public health thinking is thought to be opposed to the promotion of individual autonomy over health, but we have argued that each can help promote the other-we need to respect individuals, but in the context that every individual is part of a community or communities-a simple fact that itself helps to shape that individual's life, health and way of thinking.

It is also relevant to note in conclusion that a prerequisite for successful HIV prevention research is greater understanding of the meaning and control of sexual activity in different cultures. Without such understanding efforts to study or impose ideas about protection are likely to be less effective. Much work remains to be done to develop and implement effective preventive measures.

\section{Note}

1. The subject of the appropriate standard of care is again a much discussed subject that is beyond the breadth of this discussion. However, consideration of this dilemma is relevant for all participants and especially when considering the inclusion of minors in these trials.

\section{Acknowledgements}

We acknowledge with thanks constructive comments from Angus Dawson and anonymous reviewers. SRB's research is funded in part by the U.S. National Institutes of Health's Fogarty International Center (Grant number 1R25 TW06057), and the University of Toronto's Joint Centre for Bioethics.

\section{References}

Alliance for Microbicide Development (2008). Microbicide Candidates in Ongoing Clinical Trials. Available from http://www.global-campaign.org /candidates.htm [accessed 5 July 2008].

Anonymous. (2005). Editorial: The Trials of Tenofovir Trials. Lancet, 365, 1111.

Benatar, S. R. (1998). Global Disparities in Health and Human Rights: A Critical Commentary. American Journal of Public Health, 88, 295-300.

Benatar, S. R. (2006). Facing Ethical Challenges in Rolling Out Antiretroviral Treatment in Resource in Resource-Poor Countries: Comment on 'They Call it Patient Selection' in Khayelitsha. Cambridge Quarterly of Healthcare Ethics, 15, 322-330. 
Benatar, S. R., Daar, A. and Singer, P. A. (2003). Global Health Ethics: The Rationale for Mutual Caring. International Affairs, 79, 107-138.

Birn, A. E. (2005). Gates' Grandest Challenge: Transcending Technology as Public Health Ideology. Lancet, Published online 11 March 2005, available from http://image.thelancet.com/extras/04art6429web.pdf.

Childress, J. F., Faden, R. R., Gaare, R. D., Gostin, L. O., Kahn, J., Bonnie, R. J., Kass, N. E., Mastroianni, Moreno, J. O. and Nieburg, P. (2002). Public Health Ethics: Mapping the Terrain. Journal of Law, Medicine \& Ethics, 30, 170-178.

Epstein, S. (2007). Inclusion; The Politics of Difference in Medical Research. Chicago: University of Chicago Press.

Global Campaign for Microbicides. (2005). Rethinking the Ethical Roadmap for Clinical Testing of Microbicides: Report on an International Consultation. Available from www.global-campaign.org [accessed 21 March 2007].

International Partnership for Microbicides. 2005. Microbicides: An Essential HIV Prevention Strategy for Achieving the Millennium Development Goals. Available at http://ipm-microbicides.org/pdfs /english/ipm 'publications/2005/policy'k'092005.pdf.

Jaspan, H. B., Berwick, J. R., Myer, L., Mathews, C., Flisher, A. J., Wood, R. and Bekker, L. (2006). Adolescent HIV Prevalence, Sexual Risk, and Willingness to Participate in HIV Vaccine Trials. Journal of Adolescent Health, 39, 642-648.

Kass, N. E. (2001). An Ethics Framework for Public Health. American Journal of Public Health, 91, 1776.

Kock, A., Skoler, S., Ahmed, K. and Govender, S. (2006). Enrolling Adolescents in Research on HIV: Young Women Must Be Included. PLoS Med, 3, e357, available from http://www.pubmedcentral.nih.gov /artidlefinder.fcgi?artid=1762084 [accessed 21 March 2007].

Macqueen, K. M. and Karim, Q. A. (2007). Adolescents and HIV Clinical Trials: Ethics, Culture, and Context. Journal of the Association of Nurses in AIDS Care, 18, 78-82.

Molyneux, C. S., Wassenaar, D. R., Peshu, N. and Marsh, K. (2005). Even if they ask you to Stand by a Tree All Day, you will have to do it (laughter)....!': Community Voices on the Notion and Practice of Informed Consent for Biomedical Research in Developing Countries. Social Science and Medicine, 61, 462454.

Nixon, S., Upshur, R., Robertson, A., Benatar, S., Thompson, A. and Daar, A. (2005). Public Health Ethics. In Ries, N., Caulfield, T. and Bailey, T. (eds), Public Health $\ll$ Law Policy in Canada. Canada: Lexis Nexis, pp. 3958.

Pettifor, A. E., Rees, H. V., Kleinschmidt, I., Steffenson, A. E., MacPhail, C., Hlongwa-Madikizela, L., Vermaak, K. and Padian, N. S. (2005). Young People's Sexual Health in South Africa: HIV Prevalence and Sexual Behaviors from a Nationally Representative Household Survey. AIDS, 19, 15251534.

Roberts, M. J. and Reich, M. R. (2002). Ethical Analysis in Public Health. Lancet, 359, 1055.

Shapiro, K. and Benatar, S. R. (2005). HIV Prevention Research and Global Inequality: Steps towards Improved Standards of Care. Journal of Medical Ethics, 31, 39-47.

Shisana, O., Rehle, T., Simbayi, L. C., Parker, W., Zuma, K., Bhana, A., Connoly, C., Jooste, S. and Piillay, V. (2005). South African National HIV Incidence, HIV Prevalence, Behaviour and Communications Survey. Cape Town: HSRC Press; 2005.

Singer, P. A., Benatar, S. R., Bernstein, P., Daar, A. S., Dickens, B. M., MacRae, S. K., Upshur, R. E. G., Wright, L. and Zlotnik, Shaul R. (2003). Ethics and SARS: Lessons from Toronto. British Medical Journal, 327, 1342.

Singh, J. A., Karim, S. S. A., Karim, Q. A., Mlisana, K., Williams, C., Gray, C., Govender, M. and Gray, A. (2006). Enrolling Adolescents in Research on HIV and other Sensitive Issues: Lessons from South Africa. PLoS Med, 3 e180, available from http://medicine.plosjournals.org/perlserv/ ?request $=$ get-downument $\&$ doi $=101371$ /journal .pmed.0030180 [accessed 21 March 2007].

Skoler-Karpoff, S., Ramjee, G., Ahmed, K., Altini, L., Plagianos, M. G., Friedland, B., Govender, S., De Kock, A., Cassim, N., Palanee, T., Dozier, G., Maguire, R. and Lahteenmaki, P. (2008). Efficacy of Carraguard for Prevention of HIV Infection in Women in South Africa: A Randomised, DoubleBlind, Placebo-Controlled Trial. Lancet, 372, 19771987.

Susman, E. J., Dorn, L. D. and Fletcher, J. C. (1992). Participation in Biomedical Research: The Consent Process as Viewed by Children, Adolescents, Young Adults, and Physicians. Journal of Pediatrics, 121, 547552.

Upshur, R. E. (2002). Principles for the Justification of Public Health Intervention. Canadian Journal of Public Health, 93, 101-103.

Wheeler, R. (2006). Editorial—Gillick or Fraser? A Plea for Consistency Over Competence in Children. British Medical Journal, 332, 807. 
Copyright of Public Health Ethics is the property of Oxford University Press / UK and its content may not be copied or emailed to multiple sites or posted to a listserv without the copyright holder's express written permission. However, users may print, download, or email articles for individual use. 